\title{
Myocardial infarction detection using wavelet analysis of ECG signal
}

\author{
N S Davydov and A G Khramov ${ }^{1}$ \\ ${ }^{1}$ Samara National Research University, Moskovskoe Shosse 34, Samara, Russia, 443086
}

\begin{abstract}
The new method of ECG signal segmentation and feature extraction is developed and tested with the use of SVM classification method in this paper. The algorithm based on discrete wavelet transformation and several new steps of beat extraction is proposed in this paper. We found out that the most part of algorithms is not easy to understand and realize. During the developing we made each step of algorithm clear and valid, so anyone can realize it when it needs. The output of preprocessing method is common standard segment, which accumulate all information from ECG signal and, in fact, this segment is the trend line of cardiac cycle in ECG signal. The feature extraction method based on cross wavelet transformation is used in this paper. Cross wavelet spectrum and wavelet coherence are two characteristics which are used for feature extraction. Classification method based on Support Vector Machine and several different transformation kernels is used and sensitivity, specificity and accuracy for each algorithm is calculated. The best result is $0.87,0.8481$ and 0.8603 for sensitivity, specificity and accuracy, respectively.
\end{abstract}

\section{Introduction}

The raw signal of ECG is a time series with a specific duration and sampling frequency which depends on type of using device. The first step of ECG analysis is the preprocessing of signal. It can be skipped, but the whole signal is hard to analyzed, and it may take more time than the analysis of a common segment. Main step of signal preprocessing is cardiac cycle extraction. Discrete wavelet transformation is used in this paper for a trend line deleting, noise filtration and cycle extraction $[1,2]$.

After extraction of the set of cardiac cycles, the average standard segment is calculated. This standard segments represents all information which the signal consists. Each segment is interpolated and normalized according to the requirements of the segment model. A similar procedure is performed for each of the signals.

The next step in the analysis is the cross wavelet transformation of the standard signal segment and the template signal segment of the healthy patient. Cross wavelet transformation is based on continuous wavelet transformation and helps to find the connection between the frequencies of two input signals. It is often used in the analysis of geographic data [3]. Unlike the Fourier transform, which represents the amplitude-frequency characteristic of a signal, a continuous wavelet transformation gives a representation in the time-frequency domain. Thus, the wavelet correlation and cross wavelet spectrum obtained after the transformation make it possible to isolate the frequency-time regions of interest for distinguishing features.

There are many methods for data classification. Many of them are based on convolutional neural networks and give different results depending on the considered features. For example, the method based on shallow convolutional neural networks and the analysis of the second, third and AVF leads 
gives sensitivity, specificity and accuracy values of $0.8533,0.8409$ and 0.8454 , respectively [4]. There are also methods for classifying tree-based solutions and blind signal separation [5, 6]. However, in this paper, the method of Support Vector Machine will be used to classify the obtained features, which is also widely used in the classification of data [7]. Also in this paper, to obtain a classification result close to that which can be obtained by practical application of the algorithm, the technique of crossvalidation data is used. All signals of the electrocardiogram and patient data were taken from the database of the Massachusetts Institute of Technology.

\section{Signal preprocessing}

The first step is to bring the signal to the required view of a common standard segment is signal segmentation to cardiac cycles, which will allow working with parts containing the most important information about the patient. Segmentation is carried out using a discrete wavelet transform.

\subsection{Signal segmentation}

The discrete wavelet transform operates as follows: the input signal is analyzed in different frequency bands with different resolutions by decomposing the signal into rough approximation and details. During the transformation, two sets of functions perform their work: scaling functions and wavelets corresponding to low-frequency and high-frequency filters. The signal passes through two types of filters and the output is obtained by the coefficients of the signal decomposition using a highfrequency and low-frequency filter. At the next level, the procedure is repeated for the output of the low-pass filter, but with a lower frequency resolution and only for half the frequency band.

Thus, the time resolution of the signal is reduced by half, the time-based decimation takes place, but the frequency resolution is doubled with each level. This procedure may be performed until there are 2 counts left. For instance, a signal of 512 samples can be decomposed into 8 levels of discrete wavelet transform. As a result of the decomposition at each level, the level coefficients at the output of the high-pass filter and the additional level coefficients will be obtained at the output of the low-pass filter if further decomposition is not performed.

Input signals used in this study have a different length and amplitude. Also, each of them has a wandering trend line that affects the position of each heart cycle and can cause undesirable distortions during further processing. An example of a raw ECG signal with a present wandering trend line is shown in Figure 1.

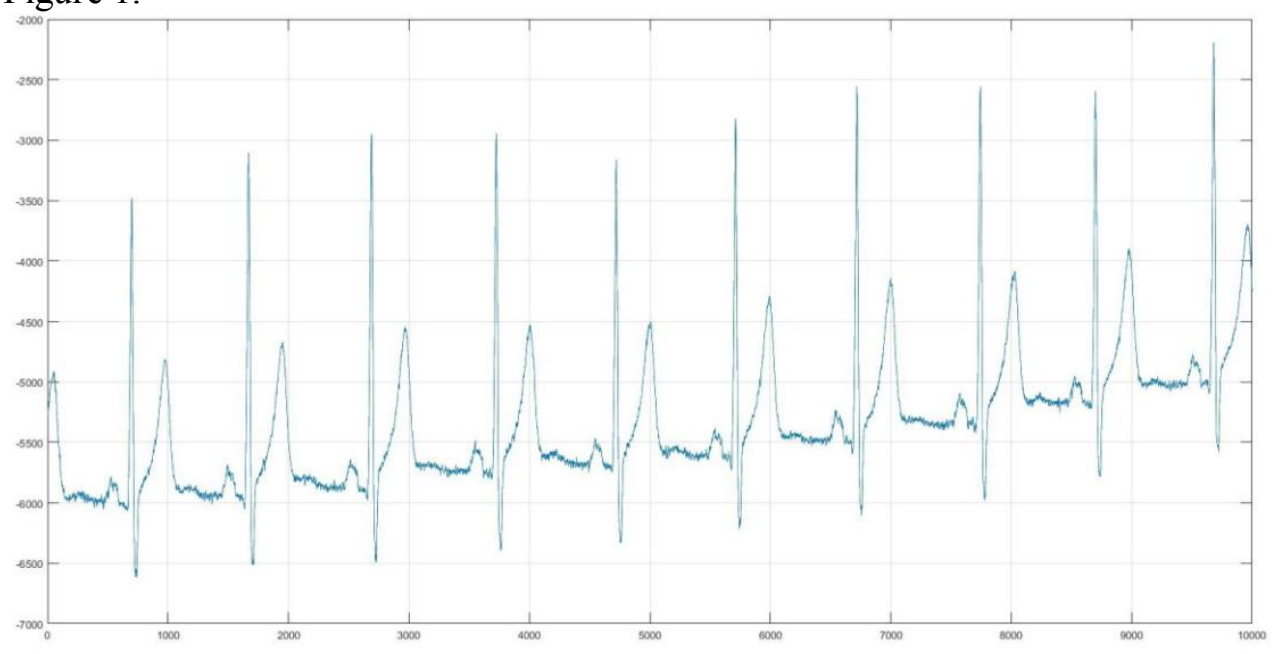

Figure 1. Raw ECG signal with a trend line.

The wandering of the trend line is associated with the physical movements of the chest in the process of patient breathing. This noise is a low-frequency physical noise and its elimination is necessary to obtain a stable signal and its subsequent processing.

The frequency range of the trend line is between 0 and $0.5 \mathrm{~Hz}$. The method used to remove the wandering trend line is based on the expansion using a discrete wavelet transform to 8 levels and the 
subsequent reconstruction of the signal at 8 levels without using the additional level coefficients. Due to the fact that the signal was restored from those coefficients that contain all the main details of the original signal without the trend line. Also, to prepare the signal for further processing, it is required to remove high-frequency noise. This can be done by decomposing the signal into 3 levels of discrete wavelet transform and restoring the signal by the coefficients of the third and additional levels, thereby removing high-frequency noise from the signal.

It is necessary to isolate the most important point of each cardiac cycle-the R-peak to perform signal segmentation. The frequency range corresponding to the QRS-complex and the R-peak is between 3 and $40 \mathrm{~Hz}$, which means that the signal decomposition into 5 levels of discrete wavelet transform and subsequent signal recovery by the coefficients of 4 and 5 levels is required. After the signal is restored, it will contain only the peak values at time positions that correspond to the $\mathrm{R}$ peaks in the original signal. Detection of peaks occurs by successively searching for a maximum with a sliding window width equal to half of the sampling rate and a maximum value threshold of at least $10 \%$ of the amplitude of the maximum point in the reconstructed signal. The result of projecting the found points to the source signal is shown in Figure 2.

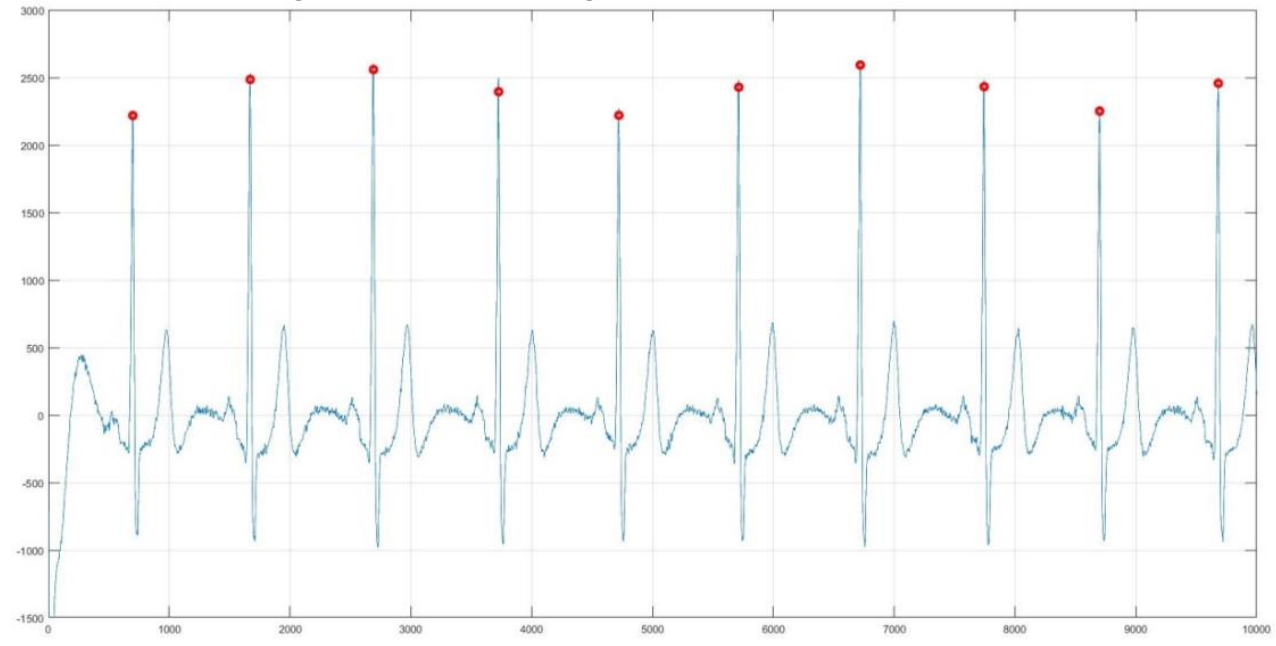

Figure 2. Detected R peaks.

The final step of the segmentation of the ECG signal will be the separation of the signal into cardiac cycles relative to the R-peak. The length of the heart cycle of each patient is different and depends on its pulse and how long the electrocardiogram is taken. In this regard, the following formula is developed to calculate the length of the patient's cardiac cycle:

$$
L=\frac{\text { LengthOfSignal }}{\text { Pulse }} \text {. }
$$

After length calculating of the heart cycle, it is necessary to isolate it relative to each R-peak. For this, an interval equal to one third of the length of the entire cycle to the left of the R-peak is taken and an interval equal to two-thirds of the length of the entire cycle to the right of the R-peak. However, there is a possibility that the first or last heart beat is not recorded completely, so the presence of this cardiac cycle will interfere with further processing of the signal. To avoid such an error, all cardiac cycles except for the first and the last detected is used in further study.

Thus, the signal is uniformly divided into cardiac cycles of the same length, but for each patient this length will be different. The whole set of segments obtained as a result of dividing the ECG signal is demonstrated in Figure 3.

\subsection{The set of cardiac segments processing}

The first thing that needs to be done to bring a set of segments to the form of a common standard segment is to interpolate all cardiac cycles and normalize each of them. As the basic requirements for the model of the common standard segment, a length of 1000 samples is chosen and the maximum Rpeak value was equal to 1 . 


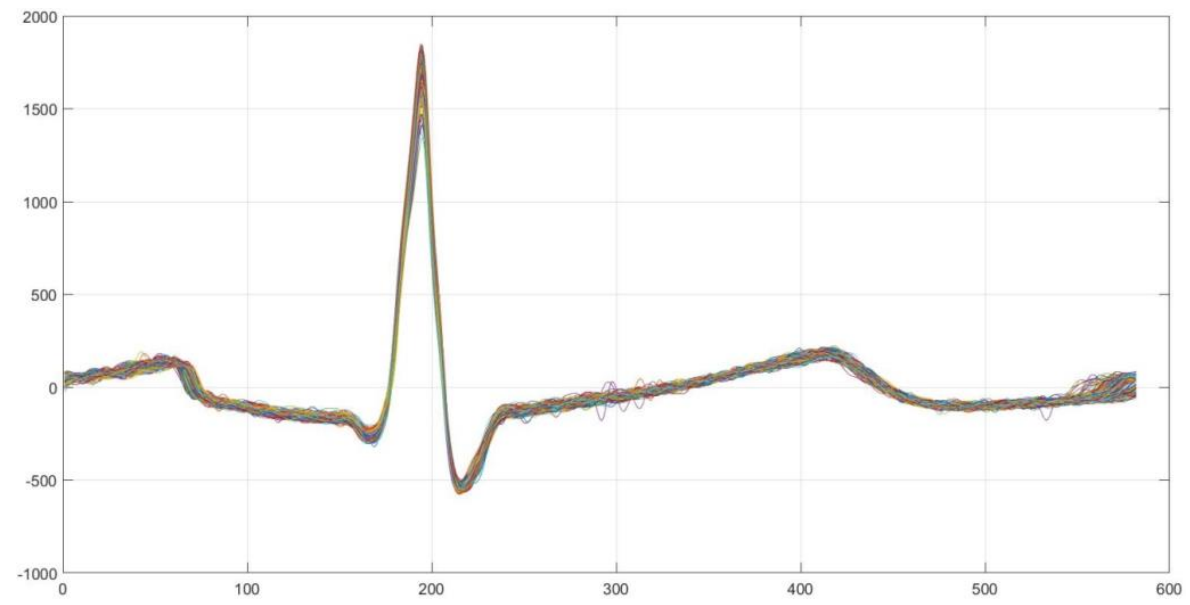

Figure 3. The set of cardiac cycles of ECG signal.

Interpolation is required in order to add points to those segments that are less than the required length and to shrink those segments that are longer than the required length. To perform this operation, we used linear interpolation of two points with fixation of the first and last reference points.

Normalization requires that all segments have the same amplitude range. The difference in the amplitude range is due to the physical characteristics of the patient's bodies and the sensitivity of the instrument.

The final step of bringing the ECG signal to the form of a common standard segment is an averaging of segments set. This procedure is necessary in order to obtain the overall shape of the entire set of segments and to drown out the high-frequency. It is worth mentioning that this step can drown erroneously selected cardiac cycles, which are not. For example, if any physical noise with frequency equal to the frequency of the QRS complex was recorded during the ECG removal, it can be defined as part of the cardiac cycle. In this regard, averaging will significantly reduce the contribution of the defective cycle to the general information segment of the signal. Also, in the case of pathological signals, various heart murmurs and heart damage can affect the waveform. However, the detection of these damages can be based on the selection of the form - the average standard segment, and the subsequent finding of the greatest deviation from this segment.

The result of final steps of signal processing is illustrated for set of segments from Figure 3 on Figure 4.

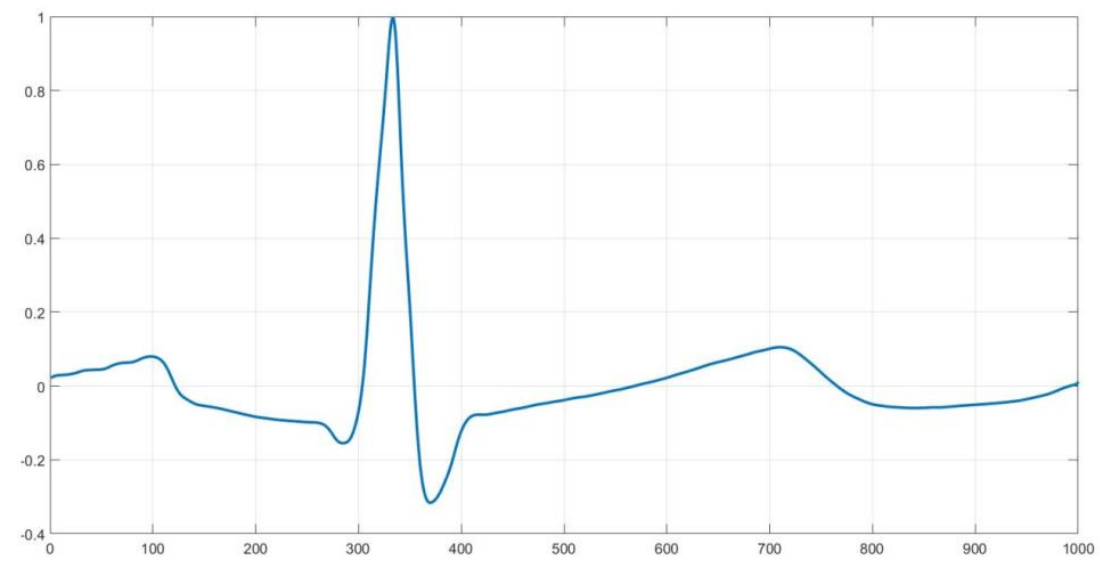

Figure 4. Common standard segment of ECG signal.

\section{Features extraction}

Continuous wavelet transformation is an efficient way to get time-frequency characteristics of input signal. Also it is possible to frequency comparation of two different signals by performing a cross wavelet transformation. The result of the cross wavelet transformation will be two characteristics: cross wavelet spectrum and wavelet coherence. 
The cross wavelet spectrum shows at what frequencies and at what time point the signals are different or similar. If the signals are different, then the image on the wavelet spectrum is darker, and in the other case lighter, which will further distinguish the signs of the difference between the forms of the common standard segment and the template. The cross wavelet spectrum of the two signals is calculated by the following formula:

$$
\Psi^{x y}=\Psi^{x} \Psi^{y *} .
$$

The wavelet coherence shows how the frequencies of the two signals under consideration are interrelated, since the wavelet transformation treats the signals precisely in the frequency-time range. The wavelet coherence of the two signals is calculated by the following formula:

$$
R_{\tau}(s)=\frac{S\left(s^{-1} \Psi_{\tau}^{x y}(s)\right)}{S\left(s^{-1}\left|\Psi_{\tau}^{x}(s)\right|\right) \cdot S\left(s^{-1}\left|\Psi_{\tau}^{y}(s)\right|\right)}
$$

To calculate features for classification, it is necessary to sum the values of the cross wavelet spectrum and wavelet coherence, thereby reducing the dimensions of the available features in the form of a spectrum and a coherence.

The first step is the summation over the time interval, which corresponds to the place of interest in the heart cycle. In the case of myocardial infarction, the QT interval is considered, which is between 250 and 750 time points. After this procedure the scale vectors of chosen time interval is taken and illustrated in Figure 5.

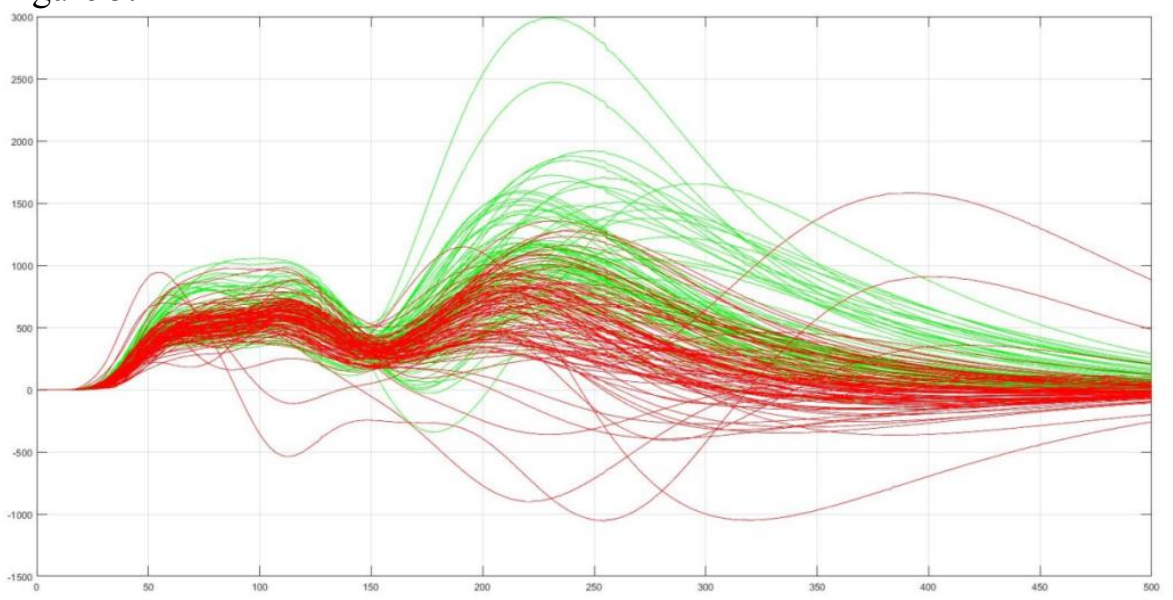

Figure 5. The scale vector of QT interval.

The next summation must go on a scale, however, the selection of the most correct interval is not obvious, therefore, an interval from 150 to 400 scale is chosen, but later, after the classifiers are formed, additional numerical experiments will be carried out in order to determine the most effective summation interval by scale. The final characteristics are formed by the following formulas:

$$
\begin{gathered}
p p=\sum_{s=150}^{400} \sum_{\tau=250}^{750} \Psi^{x y}(\tau, \mathrm{s}), \\
p a=\sum_{s=150}^{400} \sum_{\tau=250}^{750} R(\tau, \mathrm{s}) .
\end{gathered}
$$

The characteristic is a point in a two-dimensional space with coordinates $(p p, p a)$, which will allow visualizing the feature space and the classification process.

\section{Classification of extracted features}

An experimental study of the developed algorithms for signal processing and features extraction is tested with use of Support Vector Machine with a different type of hyperplane constructions and kernel functions.

The input data for the experiment are 179 ECG records with a sampling frequency of $1000 \mathrm{~Hz} .100$ records belong to patients with myocardial infarction, and 79 of them belong to healthy patients. In 
each ECG record, a signal was taken from the first standard lead, since it contains the most common information and the most common signs of myocardial infarction. All data were taken from the database of the Massachusetts Institute of Technology, provided by the Physikalisch-Technische Bundesanstalt.

Each of the records has been pre-processed, the noise is filtered out. The resulting data set was classified by three kinds of trained classifiers and cross-validated using the leave-one-out method. The result for accuracy of each classification is provided in the table 1.

Table 1. Accuracy of classification.

\begin{tabular}{cccccccc}
\hline \multirow{2}{*}{ Method } & \multicolumn{7}{c}{ Kernel functions } \\
\cline { 2 - 7 } & Linear & Quadr. & 3 order & 4 order & 5 order & 6 order & Gaussian \\
\hline QP & 0,8603 & 0,8380 & 0,8547 & 0,8492 & 0,8603 & 0,8547 & 0,8324 \\
\hline SMO & 0,8547 & 0,8324 & 0,8324 & 0,8492 & 0,8659 & 0,8547 & 0,8268 \\
\hline LS & 0,8268 & 0,8659 & 0,8380 & 0,8492 & 0,8380 & 0,8436 & 0,8380
\end{tabular}

From the obtained experimental results it is clear that the classification based on the algorithm of sequential minimal optimization (SMO) with a kernel function in form of a fifth-order polynomial and the method of least squares with a quadratic kernel turned out to be most efficient. Classification by a polynomial of the fifth order is given as an example in Figure 6.

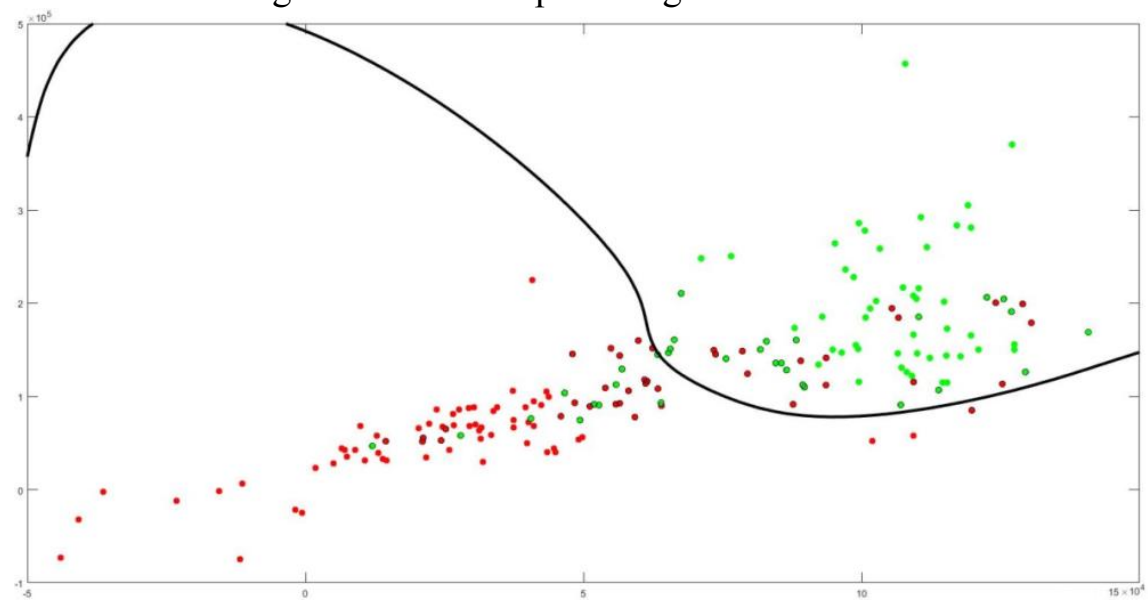

Figure 6. Classification by SVM with a fifth-order polynomial kernel.

Further, it is required to compare the obtained results with other methods of detecting myocardial infarction. It is worth noting that different articles use different methods for distinguishing signs from the ECG signal, and in some of them the entire signal is analyzed, so that a direct comparison of the result of classification with each of them is not completely correct. Also, the difference between the represented algorithms for extracting features can be that the algorithm considers only one ECG lead out of twelve, while the other algorithms are based on several leads.

The first algorithm considered for comparison is based on shallow convolutional neural networks $(\mathrm{SCNN})$, in which the entire signal in three leads is analyzed and the differences between healthy and unhealthy signals are searched. Also, in this algorithm, cross-validation of the data was carried out. The following algorithm is based on the method of blind signal separation. The problem of blind signal separation (BSS) and noise is an extremely urgent and complex mathematical task. Algorithms based on the method of support vectors have different directions. In one of the articles considered, the ECG signal is reduced to a three-dimensional form and the three-dimensional signs of the disease are calculated and the subsequent application of the multilayer support vector method (MSVM) is used. The comparison of these method in table 2 . 
Table 2. Comparison with other methods.

\begin{tabular}{cccc}
\hline Methods & Sensitivity & Specificity & Accuracy \\
\hline This study & 0,8700 & 0,8481 & 0,8603 \\
\hline SCNN & 0,8533 & 0,8409 & 0,8454 \\
\hline BSS & 0,9837 & 0,9404 & 0,9677 \\
\hline MSVM & 0,8682 & 0,9105 & 0,8543
\end{tabular}

\section{Conclusion}

In this study, a method for extracting features of myocardial infarction from an ECG signal with the use of a cross-sectional wavelet transformation was developed. As a result of the work, the features were obtained, on the basis of which classification was carried out by the method of support vector machine with different approaches to constructing a separating hyperplane and various transformation kernels. The best result was obtained using the method of support vectors, which was based on sequential minimal optimization with a transformation kernel in the form of a polynomial of the fifth degree. As a result, the sensitivity values (0.8700), specificity (0.8481) and accuracy (0.8603) were calculated. Classification was carried out using the method of cross-sectional cross-checking data to obtain the result, which most adequately shows the work of the algorithm in practical application.

As a further development of the study, it is planned to use neural networks and gradient-boosting methods to improve the result of the classification. Also during the research it was noted that taking the middle segment as a common standard is not always true. In this connection, a study will be carried out when the segment that is most different from the average is taken as the general standard segment. However, in this case, a more efficient method for filtering the signal from physical noise will be required.

\section{References}

[1] Haddadi R, Abdelmounim E and Hanine M 2014 Discrete wavelet transform based algorithm for recognition of qrs complexes World of Computer Science and Information Technology Journal 4127

[2] Chandel S and Singh K 2016 ECG denoising using wavelet transform and soft thresholding International Journal of Advanced Research in Computer Science and Software Engineering 6370

[3] Grinsted A, Moore J C and Jevrejeva S 2004 Application of the cross wavelet transform and wavelet coherence to geophysical time series Nonlinear Processes in Geophysics 11561

[4] Tahsin R and Celia S 2017 Detection of inferior myocardial infarction using shallow convolutional neural networks IEEE Region 10 Humanitarian Technology Conference 1718

[5] Khatun S and Morshed B I 2017 Detection of Myocardial Infarction and Arrhythmia from Single-Lead ECG Data using Bagging Trees Classifier Electro Information Technology 1520

[6] Devika M G and Aneesh R P 2016 Myocardial infarction fetection using hybrid BSS method International Conference on Communication Systems and Networks 1167

[7] Dhawan A, Wenzel B, George S, Gussak I, Bojovic B and Panescu D 2012 Detection of acute myocardial infarction from serial ecg using multilayer support vector machine 34th Annual International Conference of the IEEE EMBS 12704 